\title{
On Determining the Critical Path of Activity Network with Normalized Heptagonal Fuzzy Data
}

\author{
Hamiden Abd El-Wahed Khalifa $\mathbb{D}^{1,2}$ Majed G. Alharbi $\mathbb{D}^{3},{ }^{3}$ and Pavan Kumar $\mathbb{D}^{4}$ \\ ${ }^{1}$ Operations Research Department, Faculty of Graduate Studies for Statistical Research, Cairo University, Giza, Egypt \\ ${ }^{2}$ Mathematics Department, College of Science and Arts, Al-Badaya, Qassim University, Buraydah, Saudi Arabia \\ ${ }^{3}$ Mathematics Mathematics, College of Science and Arts, Methnab, Qassim University, Saudi Arabia \\ ${ }^{4}$ Mathematics Division, School of Advanced Sciences \& Languages, VIT Bhopal University, Sehore-466114, MP, India
}

Correspondence should be addressed to Pavan Kumar; pavankmaths@gmail.com

Received 5 December 2020; Accepted 18 June 2021; Published 5 July 2021

Academic Editor: Giovanni Pau

Copyright (c) 2021 Hamiden Abd El-Wahed Khalifa et al. This is an open access article distributed under the Creative Commons Attribution License, which permits unrestricted use, distribution, and reproduction in any medium, provided the original work is properly cited.

\begin{abstract}
In this research work, an approach to determine the critical path of activity network with normalized heptagonal fuzzy data is proposed. In the proposed model, we attempt to develop a method for solving litigation problems by experts when they share the same information but differ in their opinions. The concepts for a critical path method (CPM) optimization with two kinds of data are as follows: those are to be optimistic and those considered being pessimistic. A numerical example is given for the illustration of the proposed approach and gain more insights. Based on the findings of the proposed work, we observe that the floating times with optimistic data are always smaller than or equal to the corresponding floating times for the pessimistic data.
\end{abstract}

\section{Introduction}

The critical path method (CPM) is one of the most important concepts in network analysis. It is used as method for solving the complications of a project by preparing the networks and to determine the earliest date an activity can start and the earliest date can be finished. Also, it is an algorithm for scheduling a set of project activities. In addition, it is commonly used in conjunction with the program evaluation and review technique (PERT). A technique for project planning that was developed known as critical path method (CPM) was developed in 1950 by DuPont and was first used in missiledefense construction projects. When applying CPM, there are several steps that can be summarized as follows:

(i) Define the required tasks and put them down in a sequenced list

(ii) Create a flowchart or other diagram showing each task in relation to the others (iii) Identify the critical and noncritical relationships among tasks

(iv) Locate or devise alternatives for the most critical paths

CPM was emerged to support and serve as an alternative to Gantt chart. Studies and researches enumerate the possible causes of the breach to include inadequate knowledge and experience of the technique and lack of adoption of reluctance to adopt a new technological innovation as well as cost of operations [1]. Aliyu [2] clarified the effectiveness of the CPM in planning, scheduling, organizing, coordinating, managing, and controlling of project time and cost, where this study concludes that the CPM is not difficult to apply, and when applied, it improves interdepartmental communications, gives clear definition of responsibilities, and minimizes the occurrence of crisis management. Agyei [3] defined the trade-off by crashing the activities using linear programming technique and concluded with an acceptable 
cost to complete the project construction. Rautela et al. [4] concluded that the CPM can be used by every department as a tool for meeting the commitment of the delivery to other related departments and thereby reducing the problem of internal delays which causes the delay in the delivery of the final product of the company where in this case the company being a shoe production plant. Chu et al. [5] gave a new definition on the critical path in stochastic network and proposed a modified label-correcting tracing algorithm to solve it. Also, they compared the results with the Monte Carlo simulation, where the approach proposed can accurately determine the critical path in stochastic networks.

In literature, first of all, Zadeh [6] proposed the philosophy of fuzzy sets. Decision-making in a fuzzy environment has an improvement and a great help in the management decision problems [7]. Zimmermann [8] introduced fuzzy programming and linear programming with multiple objective functions. Later, several researchers worked in fuzzy set theory. Dubois and Prade [9] studied the theory and applications of fuzzy sets and systems. Kaufmann and Gupta [10] studied several fuzzy mathematical models with their applications to engineering and management sciences. Chanas and Zielinski [11] introduced the relations between the notion of fuzzy criticality and the notion of interval criticality. Also, they investigated two methods of calculations of the path degree of criticality. Arikan and Gungor [12] studied the multiple objective problem for project networks. They implemented the fuzzy goal programming to it as application point of view. Liang and Han [13] also studied the fuzzy critical path for project network. Zielinkski [14] presented their study by computing the latest starting times and floats of activities. They considered a network with imprecise durations, in their model formulation.

Chen [15] studied the project network and further investigated the critical paths under the assumption of fuzzy activity times. Chen and Hsueh [16] proposed an approach to critical path problem in project network case. They considered the fuzzy model in their proposed problem. Ghazanfari et al. [17] derived a novel solution procedure for the timecost trade-off model by implementing the fuzzy decision variables in their model. Shankar et al. [18] derived a solution method, which is based on analytical concept. They applied this method to determine the critical paths, where the critical paths were considered in a fuzzy project network by Liang [19]. Applying fuzzy goal programming to project management decisions with multiple goals in uncertain environments. [20] developed a solution procedure for the determination of total float time as well as the critical path. They considered the fuzzy uncertainty in their project network model. Ammar [21] studied the optimization of project time-cost trade-off problem by considering the assumption of discounted cash flows. Shahsavari Pour et al. [22] developed a novel methodology to solve the critical path model with the assumption of fuzzy processing time.

Elizabeth and Sujatha [23] studied the CPM. They considered the fuzzy concept for the project network in their proposed work. Khalaf [24] proposed a solution procedure for the fuzzy project scheduling problem under the assumption of a ranking function. Oladeinde and Itsisor [25] inves- tigated the CPM. Later, they applied it as application of fuzzy theory to project scheduling model. Anusuya and Balasowandari [26] discussed various measures for determining the critical path in fuzzy environment. Yao and Lin [27] investigated a novel solution method to determine the critical paths. Their solution method is found on the concept of signed distance ranking of fuzzy numbers.

Over the few decades, several researchers used different types of fuzzy numbers to formulate the mathematical models, e.g., triangular fuzzy numbers [28], hexagonal fuzzy numbers [29], and piecewise quadratic fuzzy numbers [30]. In literature, some researchers used the heptagonal fuzzy numbers in the diverse fields of mathematical modelling, e.g., fuzzy assignment problem [31] and fuzzy queuing model [32]. Radhakrishnan and Saikeerthana [33] applied the interval concepts to solve the CPM, and they performed the evaluation review technique in project network to determine the critical path and the project duration of the network.

In this paper, a CPM for the optimization with the concept of normalized heptagonal fuzzy numbers is introduced, where the process is characterized by uncertain and subjective data. The concepts for a CPM optimization with two kinds of data are as follows: those which are considered to be pessimistic and those which are considered to be optimistic. In addition, a method for solving litigation problems by experts when they share the same information but different in their opinion is outlined.

The remainder of the paper is organized as follows: Section 2 introduces some preliminaries needed in this paper. In Section 3, basic notion and CPM optimization are introduced. In Section 4, an example is given for illustration. Section 5 presents the discussion of the results. Finally, some concluding remarks are reported in Section 6.

\section{Preliminaries}

This section introduces some of basic concepts and results related to fuzzy numbers, heptagonal fuzzy numbers, and their arithmetic operations and the associated ordinary number corresponding to the heptagonal fuzzy number.

Definition 1 (see [6]). A fuzzy set $\tilde{P}$ defined on the set of real numbers $\mathbb{R}$ is said to be fuzzy numbers if its membership function

$$
\mu_{\tilde{P}}(x): \mathbb{R} \longrightarrow[0,1]
$$

possesses the following properties:

(1) $\mu_{\tilde{P}}(x)$ is an upper semicontinuous membership function

(2) $\tilde{P}$ is convex fuzzy set, i.e., $\mu_{\tilde{P}}(\delta x+(1-\delta) y) \geq \min$ $\left\{\mu_{\tilde{P}}(x), \mu_{\tilde{P}}(y)\right\} ; \forall x, y \in \mathbb{R} ; 0 \leq \delta \leq 1$

(3) $\tilde{P}$ is normal, i.e., $\exists x_{0} \in \mathbb{R}$ for which $\mu_{\tilde{P}}\left(x_{0}\right)=1$

(4) $\operatorname{Supp}(\tilde{P})=\left\{x \in \mathbb{R}: \mu_{\tilde{P}}(x)>0\right\}$ is the support of $\tilde{P}$, and the closure $\operatorname{cl}(\operatorname{Supp}(\tilde{P}))$ is compact set 
Definition 2 (see [31]). A fuzzy number $\tilde{A}_{H}\left(\mathrm{a}_{1}, \mathrm{a}_{2}, \mathrm{a}_{3}, \mathrm{a}_{4}, \mathrm{a}_{5}\right.$, $\left.a_{6}, a_{7}\right)$ is a heptagonal fuzzy number (H.F.N.), whereas $a_{1}$, $a_{2}, a_{3}, a_{4}, a_{5}, a_{6}, a_{7} \in \mathbb{R}$ and its membership function are defined by

$$
\mu_{\tilde{A}_{H}}(x)= \begin{cases}\frac{1}{3}\left(\frac{x-a_{1}}{a_{2}-a_{1}}\right) & \text { for } a_{1} \leq x \leq a_{2}, \\ \frac{1}{3}+\frac{1}{3}\left(\frac{x-a_{2}}{a_{3}-a_{2}}\right) & \text { for } a_{2} \leq x \leq a_{3}, \\ \frac{2}{3}+\frac{1}{3}\left(\frac{x-a_{3}}{a_{4}-a_{3}}\right) & \text { for } a_{3} \leq x \leq a_{4}, \\ 1-\frac{1}{3}\left(\frac{x-a_{4}}{a_{5}-a_{4}}\right) & \text { for } a_{4} \leq x \leq a_{5}, \\ \frac{2}{3}-\frac{1}{3}\left(\frac{x-a_{5}}{a_{6}-a_{5}}\right) & \text { for } a_{5} \leq x \leq a_{6}, \\ \frac{1}{3}\left(\frac{a_{7}-x}{a_{7}-a_{6}}\right) & \text { for } a_{6} \leq x \leq a_{7}, \\ 0, \text { for } x<a_{1} \text { and } x>a_{7} .\end{cases}
$$

A H.F.N. can be characterized by the so called interval of confidence at level $\alpha$ as follows:

$$
\begin{aligned}
\tilde{A}_{H \alpha}(x)= & \left\{x \in X: \mu_{\tilde{A}_{H}} \geq \alpha\right\} \\
= & \begin{cases}{\left[P^{-}(\alpha), P^{+}(\alpha)\right]} & \text { for } \alpha \in\left[0, \frac{1}{3}\right], \\
{\left[Q^{-}(\alpha), Q^{+}(\alpha)\right]} & \text { for } \alpha \in\left[\frac{1}{3}, \frac{2}{3}\right], \\
{\left[R^{-}(\alpha), R^{+}(\alpha)\right]} & \text { for } \alpha \in\left[\frac{2}{3}, 1\right] .\end{cases}
\end{aligned}
$$

Definition 3 (see [31]). If $P^{-}(\alpha)=\alpha$ and $P^{+}(u)=\alpha$. Then, the $\alpha$-cut of $\mu_{\tilde{A}_{H}}$ is defined as follows:

$$
\begin{aligned}
{\left[P^{-}(\alpha), P^{+}(\alpha)\right]=[} & 3 \alpha\left(a_{2}-a_{1}\right)+a_{1},-3 \alpha\left(a_{7}-a_{6}\right) \\
& \left.+a_{7}\right] \text { for } \alpha \in\left[0, \frac{1}{3}\right], \\
{\left[Q^{-}(\alpha), Q^{+}(\alpha)\right]=\left[3\left(\alpha-\frac{1}{3}\right)\left(a_{3}-a_{2}\right)+a_{2},-3\left(\alpha-\frac{2}{3}\right)\right.} & \left.\cdot\left(a_{6}-a_{5}\right)+a_{5}\right] \text { for } \alpha \in\left[\frac{1}{3}, \frac{2}{3}\right], \\
R^{-}(\alpha), R^{+}(u)= & {\left[3\left(\alpha-\frac{2}{3}\right)\left(a_{4}-a_{3}\right)+a_{3},-3(\alpha-1)\right.} \\
& \left.\cdot\left(a_{5}-a_{4}\right)+a_{4}\right] \text { for } \alpha \in\left[\frac{2}{3}, 1\right] .
\end{aligned}
$$

Definition 4 (see [31]). Let $\tilde{A}_{H}=\left(a_{1}, a_{2}, a_{3}, a_{4}, a_{5}, a_{6}, a_{7}\right)$ and $\tilde{B}_{H}=\left(b_{1}, b_{2}, b_{3}, b_{4}, b_{5}, b_{6}, b_{7}\right) ; \forall a_{1}, a_{2}, a_{3}, a_{4}, a_{5}, a_{6}, a_{7} ; b_{1}$, $b_{2}, b_{3}, b_{4}, b_{5}, b_{6}, b_{7} \in \mathbb{R}, a_{1} \leq a_{2} \leq a_{3} \leq a_{4} \leq a_{5} \leq a_{6} \leq a_{7} ; b_{1} \leq$ $b_{2} \leq b_{3} \leq b_{4} \leq b_{5} \leq b_{6} \leq b_{7}$ be two HFNs. Then,

$$
\begin{aligned}
& \tilde{A}_{H \alpha}(x) \oplus \tilde{B}_{H \alpha}(x) \\
& =\left\{\begin{array}{l}
{\left[3 \alpha\left(a_{2}-a_{1}\right)+a_{1},-3 \alpha\left(a_{7}-a_{6}\right)+a_{7}\right] \text { for } \alpha \in\left[0, \frac{1}{3}\right],} \\
{\left[3\left(\alpha-\frac{1}{3}\right)\left(a_{3}-a_{2}\right)+a_{2},-3\left(\alpha-\frac{2}{3}\right)\left(a_{6}-a_{5}\right)+a_{5}\right] \text { for } \alpha \in\left[\frac{1}{3}, \frac{2}{3}\right],} \\
{\left[3\left(\alpha-\frac{2}{3}\right)\left(a_{4}-a_{3}\right)+a_{3},-3(\alpha-1)\left(a_{5}-a_{4}\right)+a_{4}\right] \text { for } \alpha \in\left[\frac{2}{3}, 1\right]}
\end{array}\right.
\end{aligned}
$$

Definition 5 (see [31]). Let $\tilde{A}_{H}=\left(a_{1}, a_{2}, a_{3}, a_{4}, a_{5}, a_{6}, a_{7}\right)$ and

$$
\tilde{B}_{H}=\left(b_{1}, b_{2}, b_{3}, b_{4}, b_{5}, b_{6}, b_{7}\right) \text {. }
$$

Then

$$
\text { Addition : } \begin{aligned}
\tilde{A}_{H} \oplus \tilde{B}_{H}= & \left(a_{1}, a_{2}, a_{3}, a_{4}, a_{5}, a_{6}, a_{7}\right) \\
& \bigoplus\left(b_{1}, b_{2}, b_{3}, b_{4}, b_{5}, b_{6}, b_{7}\right) \\
= & \left(a_{1}+b_{1}, a_{2}+b_{2}, a_{3}+b_{3}, a_{4}\right. \\
& \left.+b_{4}, a_{5}+b_{5}, a_{6}+b_{6}, a_{7}+b_{7}\right),
\end{aligned}
$$

Subtraction : $\tilde{A}_{H} \ominus \tilde{B}_{H}=\left(a_{1}, a_{2}, a_{3}, a_{4}, a_{5}, a_{6}, a_{7}\right)$

$$
\begin{aligned}
& \ominus\left(b_{1}, b_{2}, b_{3}, b_{4}, b_{5}, b_{6}, b_{7}\right) \\
= & \left(a_{1}-b_{7}, a_{2}-b_{6}, a_{3}-b_{5}, a_{4}\right. \\
& \left.-b_{4}, a_{5}-b_{3}, a_{6}-b_{2}, a_{7}-b_{1}\right),
\end{aligned}
$$

Scalar multiplication : $k \tilde{A}_{H}$

$$
=\left\{\begin{array}{l}
k\left(a_{1}, a_{2}, a_{3}, a_{4}, a_{5}, a_{6}, a_{7}\right), k \geq 0, \\
k\left(a_{7}, a_{6}, a_{5}, a_{4}, a_{3}, a_{2}, a_{1}\right), k<0 .
\end{array}\right.
$$

Definition 6. The associated ordinary (crisp) number corresponding to the H.F.N.

$\tilde{A}_{H}=\left(a_{1}, a_{2}, a_{3}, a_{4}, a_{5}, a_{6}, a_{7}\right)$ is defined by

$$
\widehat{A}_{H}=\frac{a_{1}+a_{2}+a_{3}+2 a_{4}+a_{5}+a_{6}+a_{7}}{8} .
$$

2.1. Notations. $\tilde{A}_{H}^{(i)}$ : Major of the sheaf $X^{(i)}$.

$\tilde{A}_{H}^{(i)}$ 。: Minor of the sheaf $X^{(i)}$

$\widehat{A}_{H}$ : Associated ordinary number

$(-)$ : Minkowski's subtraction

\section{Problem Formulation}

Let us introduce some basic notion needed in the problem statement.

Let us consider a sheaf $X$ composed of H.F.N.s $\tilde{A}_{H}, i=$ $1,2, \cdots, m$. Define $\tilde{A}_{H}^{\circ}$ as a "major H.F.N., as a major H.F.N." in the case of H.F.N. dominates all the other $\tilde{A}_{H i}$ 


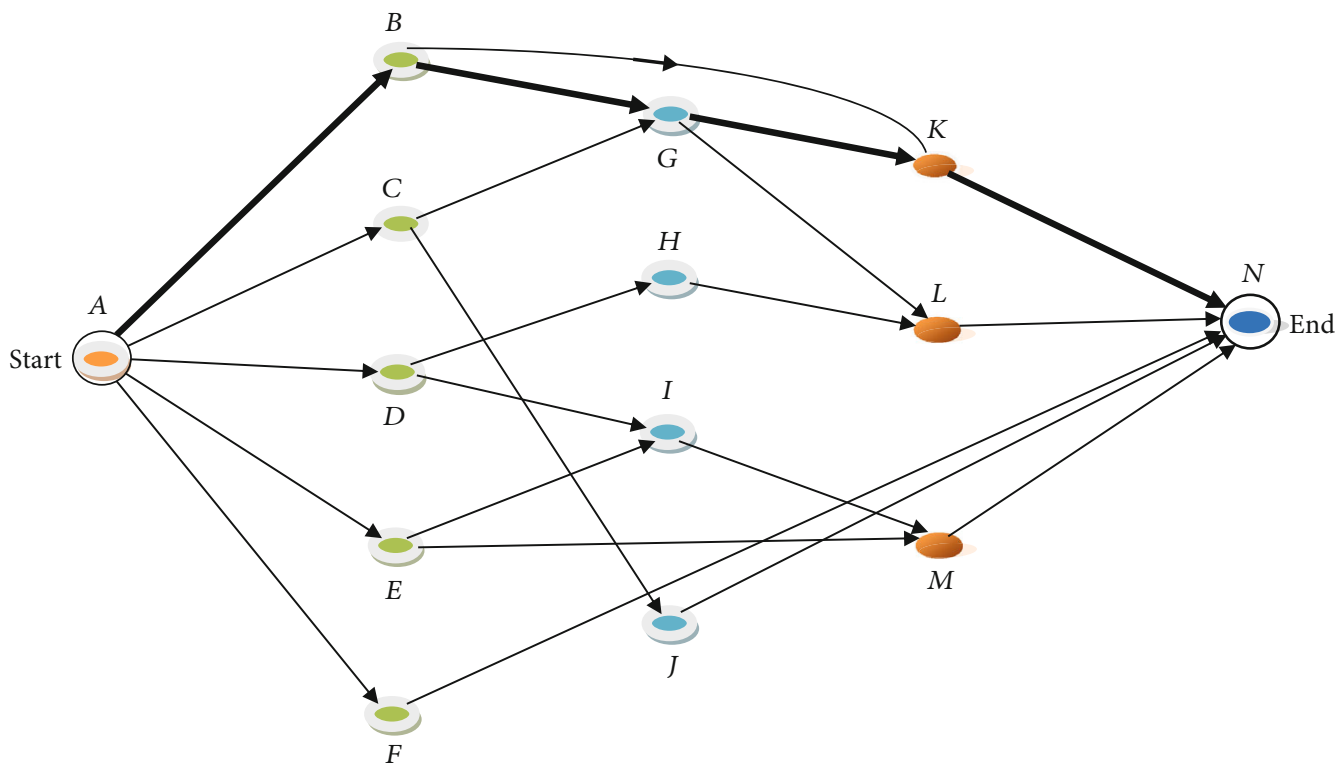

FIgURE 1: A critical path method (CPM) network.

in the sheaf $X$. The criterion of dominance is one of the following three as follows:

(i) The greatest associated ordinary number is presented by

$$
\widehat{A}_{H}^{\circ}=\left(\frac{a_{1}+a_{2}+a_{3}+2 a_{4}+a_{5}+a_{6}+a_{7}}{8}\right)^{\circ} .
$$

(ii) If criterion (i) does not separate the two H.F.N.s, those which have the best maximal presumption (the mode) will be chosen

(iii) If criteria (i) and (ii) do not separate the two H.F.N.s, the divergence will be used as a third criterion

Mathematically, the earliest time $E_{s_{j}}$ can be calculated as

$$
E_{s_{j}}=\max _{i<j}\left(E_{s_{i}}+t_{i j}\right), j>i, E_{s_{1}}=0
$$

where $t_{i j}$ is the time of the activity $(i, j)$.

Similarly, the latest time $L_{c_{i}}$ can be calculated as

$$
L_{c_{i}}=\min _{j<i}\left(L_{c_{j}}-t_{i j}\right), j<i .
$$

The critical path is at the events for which the time is zero

$$
E_{s_{j}}=L_{c_{i}}, i=1, n
$$

On the contrary, we shall call H.F.N. $\tilde{A}_{H}$ 。 a "minor H.F.N." if the H.F.N. is dominated by all others in the sheaf $M$ using, respectively, the criteria (i), (ii), and (iii), respectively.
Theorem 7 (see [10]). By using the criterion $\widehat{A}_{H}$ in (i), if $\tilde{A}_{H}^{(1)}$ 。 is the major of the sheaf $X^{(1)}$, and $\tilde{A}_{H}^{(2)}{ }^{\circ}$ is the major of the sheaf $X^{(2)}$, then the major of the sheaf $X^{(1)} \oplus X^{(2)}$ and

$$
\tilde{A}_{H}^{(1) \circ} \bigoplus \tilde{A}_{H}^{(2) \circ}=\text { major of the sheaf }\left\{X^{(1)} \text { and } X^{(2)}\right\} \text {. }
$$

Proof. (see [10]).

Definition 8 (see [10]). Minkowski's subtraction of two H.F.N.s. $\tilde{A}_{H}$ and $\tilde{B}_{H}$ is defined as

$$
\begin{aligned}
\tilde{A}_{H}(-) \tilde{B}_{H}= & \left(a_{1}, a_{2}, a_{3}, a_{4}, a_{5}, a_{6}, a_{7}\right) \underset{m}{(-)} \\
& \cdot\left(b_{1}, b_{2}, b_{3}, b_{4}, b_{5}, b_{6}, b_{7}\right) \\
= & \left(a_{1}-b_{1}, a_{2}-b_{2}, a_{3}-b_{3}, a_{4}\right. \\
& \left.-b_{4}, a_{5}-b_{5}, a_{6}-b_{6}, a_{7}-b_{7}\right) .
\end{aligned}
$$

\section{Numerical Example}

Assume the implementation of a plant has 14 vertices and it needs 22 main operations each one represented by a direct link as in the following graph (Figure 1).

For each operation on graph, four experts were interviewed, and these experts expressed their valuations in the form of H.F.N.s. Using these data, we shall compute a pessimistic forecast with a H.F.N. major and an optimistic forecast with a H.F.N. minor. The computations for both will require the maximization of the total durations until the plant will be ready for production. Table 1 gives the H.F.N. data for each link and shows the minor $(m)$ and major $(M)$ with $\mathrm{a}^{\circ}$ as a sub- or superscript, respectively. The last column gives the maximization at each vertex. When the first criterion dealing with the associated ordinary number is not sufficient, then the second and if needed the third criteria are applied. 
TABLE 1: Expert's H.F.N. and associated ordinary number for each operation from $A$ to $N$ in Figure 2.

\begin{tabular}{|c|c|c|}
\hline Operation & $\begin{array}{c}\text { Expert's H.F.N. } \\
\tilde{A}_{H}\left(a_{1}, a_{2}, a_{3}, a_{4}, a_{5}, a_{6}, a_{7}\right)\end{array}$ & $\begin{array}{c}\text { Associated ordinary number } \\
\widehat{A}_{H}^{\circ}=\left(a_{1}+a_{2}+a_{3}+2 a_{4}+a_{5}+a_{6}+a_{7}\right) / 8\end{array}$ \\
\hline $1^{\mathrm{AB}}$ & $\begin{array}{c}(3,4,5,7,9,10,11) \circ \longleftarrow m \\
(3,4,6,7,11,12,13) \\
(2,3,4,8,13,14,15)^{\circ} \longleftarrow M \\
(1,2,3,6,14,15,16)\end{array}$ & $\begin{array}{c}\frac{(3+4+5+14+9+10+11)}{8}=7 \\
7.875 \\
8.375^{\circ} \\
7.875\end{array}$ \\
\hline $2^{\mathrm{AC}}$ & $\begin{array}{l}(1,2,3,8,9,11,13)^{\circ} \longleftarrow M \\
(1,2,4,5,11,12,13) \\
(0,1,2,7,8,13,14) \\
(0,1,3,4,12,13,14)_{\circ} \longleftarrow m\end{array}$ & $\frac{(1+2+3+16+9+11+13)}{8}=6.875^{\circ}$ \\
\hline $3^{\mathrm{AD}}$ & $\begin{array}{c}(0,1,2,4,6,7,8) \circ \longleftarrow \\
(0,1,2,6,7,8,9) \\
(0,1,2,6,8,10,11)^{\circ} \longleftarrow M \\
\quad(0,1,3,4,9,10,11)\end{array}$ & $\begin{array}{c}\frac{(0+1+2+8+6+7+8)}{8}=4 \text { 。 } \\
4.875 \\
5.5^{\circ} \\
5.25\end{array}$ \\
\hline $4^{\mathrm{AE}}$ & $\begin{array}{c}(1,2,5,11,12,13,14) \\
(1,3,6,13,15,16,17)^{\circ} \longleftarrow \\
(1,2,4,10,11,12,13)_{\circ} \longleftarrow \\
\quad(1,2,5,12,13,14,15)\end{array}$ & $\frac{(1+2+5+22+12+13+14)}{8}=8.625$ \\
\hline $5^{\mathrm{AF}}$ & $\begin{array}{l}(1,2,6,12,13,14,15)^{\circ} \longleftarrow M \\
(1,2,3,5,14,15,16) \\
(1,2,5,10,12,14,17) \\
(0,1,2,8,10,13,14) 。 m\end{array}$ & $\frac{(1+2+6+24+13+14+15)}{8}=9.375^{\circ}$ \\
\hline $6^{\text {BG }}$ & $\begin{array}{c}(1,2,11,13,14,15,16) \\
(1,6,11,12,15,16,17)^{\circ} \longleftarrow M \\
(1,2,7,8,9,10,13) \circ m \\
(3,5,7,8,9,10,11)\end{array}$ & $\frac{(1+2+11+26+14+15+16)}{8}=10.625$ \\
\hline $7^{\mathrm{BK}}$ & $\begin{array}{l}(3,4,5,11,19,20,22)^{\circ} \longleftarrow M \\
(3,5,10,12,13,15,16) \\
(2,5,8,10,11,12,13) \text { 。 } \\
\quad(1,5,7,10,12,13,15)\end{array}$ & $\frac{(3+4+5+22+19+21+22)}{8}=11.875^{\circ}$ \\
\hline $8^{\mathrm{CG}}$ & $\begin{array}{c}(1,3,5,8,9,10,11) \longleftarrow m \\
(3,5,6,7,10,11,12) \\
(2,3,4,8,14,15,16)^{\circ} \longleftarrow \\
(1,3,4,8,10,12,13)\end{array}$ & $\frac{(1+3+5+16+9+10+11)}{8}=6.875$ \\
\hline $9^{\mathrm{CJ}}$ & $\begin{array}{c}(3,5,8,13,14,15,16)^{\circ} \longleftarrow M \\
(1,5,7,11,12,13,14) \\
(1,5,8,10,9,11,13) \\
(1,2,5,9,10,12,14) 。 m\end{array}$ & $\begin{array}{c}\frac{(3+5+8+26+14+15+16)}{8} \\
9.25 \\
8.375 \\
7.75 \text { 。 }\end{array}$ \\
\hline $10^{\mathrm{DH}}$ & $\begin{array}{c}(0,1,2,3,5,6,8) \\
(0,1,2,3,4,5,7) \circ m \\
(0,1,2,3,4,5,8) \\
(1,2,3,4,5,8,9)^{\circ} \longleftarrow M\end{array}$ & $\frac{(0+1+2+6+5+6+8)}{8}=3.5$ \\
\hline 11 & $\begin{array}{c}(1,2,3,4,5,7,8)_{\circ} \longleftarrow m \\
(1,2,4,5,6,7,9) \\
(0,1,2,5,6,8,9) \\
(1,3,5,6,8,9,11)^{\circ} \longleftarrow M\end{array}$ & $\frac{(1+2+3+8+5+7+8)}{8}=4.25$ 。 \\
\hline
\end{tabular}


TABle 1: Continued.

\begin{tabular}{|c|c|c|}
\hline Operation & $\begin{array}{c}\text { Expert's H.F.N. } \\
\tilde{A}_{H}\left(a_{1}, a_{2}, a_{3}, a_{4}, a_{5}, a_{6}, a_{7}\right)\end{array}$ & $\begin{array}{c}\text { Associated ordinary number } \\
\widehat{A}_{H}^{\circ}=\left(a_{1}+a_{2}+a_{3}+2 a_{4}+a_{5}+a_{6}+a_{7}\right) / 8\end{array}$ \\
\hline $12^{\mathrm{EI}}$ & $\begin{array}{c}(3,7,9,13,15,16,18)^{\circ} \longleftarrow M \\
(3,5,10,12,13,14,16) \\
(2,5,8,10,11,12,13) \longleftarrow m \\
(4,6,7,9,12,13,14)\end{array}$ & $\frac{(3+7+9+26+15+16+18)}{8}=11.75^{\circ}$ \\
\hline $13^{\mathrm{EM}}$ & $\begin{array}{c}(5,10,15,22,23,24,25)^{\circ} \longleftarrow M \\
(4,10,12,17,18,19,21) \\
(3,10,12,13,14,16,17) \\
(3,6,8,10,11,12,13) 。 m\end{array}$ & $\frac{(5+10+15+44+23+24+25)}{8}=18.25^{\circ}$ \\
\hline $14^{\mathrm{FN}}$ & $\begin{array}{c}(1,2,3,4,5,6,8) \\
(1,3,4,5,7,8,10)^{\circ} \longleftarrow M \\
(0,1,2,3,4,5,6) 。 \\
(1,2,3,4,5,6,7)\end{array}$ & $\frac{(1+2+3+8+5+6+8)}{8}=4.125$ \\
\hline $15^{\mathrm{GK}}$ & $\begin{array}{c}(11,13,14,15,16,17,18) \\
(7,10,13,16,17,18,19)_{\circ} \longleftarrow \mathrm{m} \\
(13,15,18,20,23,24,26)^{\circ} \longleftarrow \mathrm{M} \\
(10,12,15,20,21,22,23)\end{array}$ & $\frac{(11+13+14+30+16+17+18)}{8}=14.875$ \\
\hline $16^{\mathrm{GL}}$ & $\begin{array}{l}(3,5,6,7,8,9,10)^{\circ} \longleftarrow M \\
(3,4,5,6,7,8,9) \\
(5,5,5,5,5,5,5)_{\circ} \longleftarrow m \\
(5,5,5,5,5,5,5)_{\circ} \longleftarrow m\end{array}$ & $\begin{array}{c}\frac{(3+5+6+14+8+9+10)}{8} \\
6 \\
5 \\
5\end{array}$ \\
\hline${ }_{17}^{\mathrm{HL}}$ & $\begin{array}{c}(6,8,9,10,11,12,13) \\
(5,7,8,9,10,11,12) \longleftarrow m \\
(7,8,9,10,11,12,13) \\
(8,9,10,11,12,13,15)^{\circ} \longleftarrow M\end{array}$ & $\frac{(6+8+9+20+11+12+13)}{8}=9.875$ \\
\hline $18^{\mathrm{IM}}$ & $\begin{array}{c}(6,6,6,6,6,6,6) \\
(6,6,6,6,6,6,6) \\
(6,6,6,6,7,7,7)^{\circ} \longleftarrow M \\
(5,5,5,5,5,5,5) 。 m\end{array}$ & $\frac{(6+6+6+12+6+6+6)}{8}=6$ \\
\hline $19^{\mathrm{JN}}$ & $\begin{array}{c}(3,4,5,6,7,8,9)^{\circ} \longleftarrow M \\
(1,3,4,5,6,7,8) \circ M \\
(1,2,3,6,7,8,9) \\
(2,3,4,5,6,7,8)\end{array}$ & $\frac{(3+4+5+12+7+8+9)}{8}=5.75^{\circ}$ \\
\hline $20^{\mathrm{KN}}$ & $\begin{array}{c}(3,5,7,8,9,10,11) \\
(6,7,8,9,10,11,12)^{\circ} \longleftarrow M \\
(3,4,5,6,7,8,9)_{\circ} \longleftarrow m \\
(4,6,7,9,10,11,12)\end{array}$ & $\begin{array}{c}\frac{(3+5+7+16+9+10+11)}{8}=7.625 \\
9^{\circ} \\
6 。 \\
8.5\end{array}$ \\
\hline 21 & $\begin{array}{l}(0,1,2,3,4,5,6)_{\circ} \longleftarrow m \\
(2,3,4,5,6,7,8)^{\circ} \longleftarrow M \\
(4,4,4,4,4,4,4) \\
(1,2,3,5,6,7,8)\end{array}$ & $\begin{array}{c}\frac{(0+1+2+6+4+5+6)}{8}=3 \text { 。 } \\
5 \\
4 \\
4.625\end{array}$ \\
\hline $22^{\mathrm{MN}}$ & $\begin{array}{c}(5,7,8,9,10,11,12) \\
(4,6,7,9,10,11,12) \\
(5,6,7,8,9,10,11) 。 \text { } \longleftarrow m \\
(7,8,9,11,12,13,14)^{\circ} \longleftarrow M\end{array}$ & $\frac{(5+7+8+18+10+11+12)}{8}=8.875$ \\
\hline
\end{tabular}




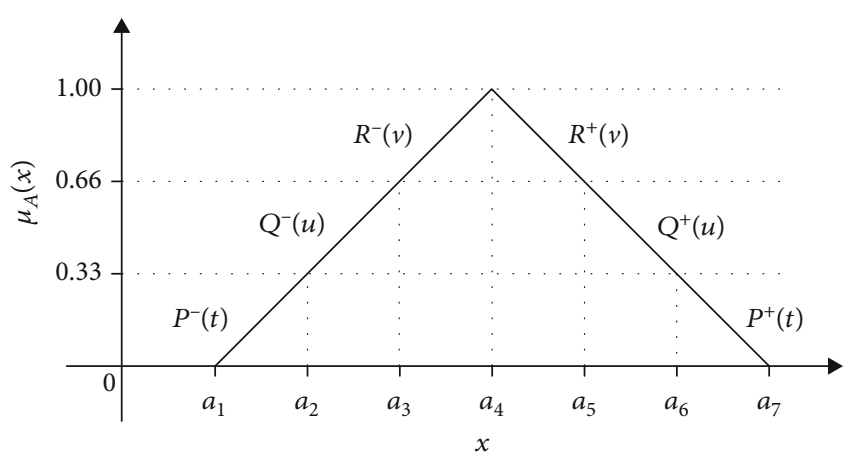

Figure 2: Graphical representation of a normal H.F.N. [31].

For each operation in Table 1, row 1 gives the H.F.N. of expert 1, row 2 that for expert 2, etc. The symbol $M$ represents the major and $m$ the minor H.F.N. The position of these ${ }^{\circ}$ has the same meaning. With these pessimistic H.F.N.s. in the next Table 2, we shall seek the optimal or critical path from start $(A)$ to the end $(N)$.

4.1. Pessimistic Critical Path Computations. The majors from the starting point $A$ to the various succeeding nodes are given by

$$
\begin{aligned}
& \longrightarrow \text { on } B:(2,3,4,8,13,14,15), \\
& \longrightarrow \text { on } C:(1,2,3,8,9,11,13), \\
& \longrightarrow \text { on } D:(0,1,2,6,8,10,11), \\
& \longrightarrow \text { on } E:(1,3,6,13,15,16,17), \\
& \longrightarrow \text { on } F:(1,2,6,12,13,14,15) .
\end{aligned}
$$

Now, we calculate the cost to arrive at the node $G$ as $B$ $\oplus B G=(2,3,4,8,13,14,15) \oplus(1,6,11,12,15,16,17)=(3$, $9,15,20,28,30,32)$ gives $(3+9+15+40+28+30+32) / 8$ $=19.625 ; \quad C \oplus C G=(1,2,3,8,9,11,13) \oplus(2,3,4,8,14,15$, 16) $=(3,5,7,16,23,26,29)$ gives $(3+5+7+32+23+26+$ 29) $/ 8=15.625$. at $G$

Therefore, the pessimistic forecast is given by the major

$$
\longrightarrow \text { on } G:(3,9,15,20,28,30,32) \text {. }
$$

We continue the computation for subsequent nodes as follows:

For $\mathbf{H}$

$$
\begin{aligned}
D \oplus D H & =(0,1,2,6,8,10,11) \oplus(1,2,3,4,5,8,9) \\
& =(1,3,5,10,13,18,20), \\
& \longrightarrow \text { on } H:(1,3,5,10,13,18,20) .
\end{aligned}
$$

Table 2: Pessimistic H. F.N. corresponding to major H. F.N. in Table 1 for each operation from $A$ to $N$ and the corresponding associated numbers.

\begin{tabular}{lcc}
\hline Path & H.F.N. $\tilde{A}_{H}^{\circ}$ & $\widehat{A}_{H}^{\circ}$ \\
\hline $1 \mathrm{AB}$ & $(2,3,4,8,13,14,15)$ & 8.375 \\
$2 \mathrm{AC}$ & $(1,2,3,8,9,11,13)$ & 6.875 \\
$3 \mathrm{AD}$ & $(0,1,2,6,8,10,11)$ & 5.5 \\
$4 \mathrm{AE}$ & $(1,3,6,13,15,16,17)$ & 10.5 \\
$5 \mathrm{AF}$ & $(1,2,6,12,13,14,15)$ & 9.375 \\
$6 \mathrm{BG}$ & $(1,6,11,12,15,16,17)$ & 11.25 \\
$7 \mathrm{BK}$ & $(3,4,5,11,19,20,22)$ & 11.875 \\
$8 \mathrm{CG}$ & $(2,3,4,8,14,15,16)$ & 8.75 \\
$9 \mathrm{CJ}$ & $(3,5,8,13,14,15,16)$ & 10.875 \\
$10 \mathrm{DH}$ & $(1,2,3,4,5,8,9)$ & 4.5 \\
$11 \mathrm{DI}$ & $(1,3,5,6,8,9,11)$ & 6.125 \\
$12 \mathrm{EI}$ & $(3,7,9,13,15,16,18)$ & 11.75 \\
$13 \mathrm{EM}$ & $(5,10,15,22,23,24,25)$ & 18.25 \\
$14 \mathrm{FN}$ & $(1,3,4,5,7,8,10)$ & 5.375 \\
$15 \mathrm{GK}$ & $(13,15,18,20,23,24,26)$ & 19.875 \\
$16 \mathrm{GL}$ & $(3,5,6,7,8,9,10)$ & 6.875 \\
$17 \mathrm{HL}$ & $(8,9,10,11,12,13,15)$ & 11.125 \\
$18 \mathrm{IM}$ & $(6,6,6,6,7,7,7)$ & 6.375 \\
$19 \mathrm{JN}$ & $(6,7,8,9,10,11,12)$ & 5.75 \\
$20 \mathrm{KN}$ & $(6,7,8,9,10,11,12)$ & 9.625 \\
$21 \mathrm{LN}$ & $(2,3,4,5,6,7,8)$ & \\
$22 \mathrm{MN}$ & $(7,8,9,11,12,13,14)$ & \\
\hline & &
\end{tabular}

For I

$$
\begin{aligned}
D \oplus \mathrm{DI} & =(0,1,2,6,8,10,11) \oplus(1,3,5,6,8,9,11) \\
& =(1,4,7,12,16,19,22), \\
E \oplus \mathrm{EI} & =(1,3,6,13,15,16,17) \oplus(3,7,9,13,15,16,18) \\
& =(4,10,15,26,30,32,35),
\end{aligned}
$$

$\longrightarrow$ on $I:(4,10,15,26,30,32,35)$ (by domination). ForJ

$$
\begin{aligned}
C \oplus \mathrm{CJ}= & (1,2,3,8,9,11,13) \oplus(3,5,8,13,14,15,16) \\
= & (4,7,11,21,23,26,29), \\
& \longrightarrow \text { on } J:(4,7,11,21,23,26,29) .
\end{aligned}
$$


For $\mathbf{K}$

$$
\begin{aligned}
G \oplus \mathrm{GK}= & ((3,9,15,20,28,30,32)) \\
& \oplus(13,15,18,20,23,24,26) \\
= & (16,24,33,40,51,54,58), \\
B \oplus \mathrm{BK}= & (2,3,4,8,13,14,15) \\
& \oplus(3,4,5,11,19,20,22) \\
= & (5,7,9,19,32,34,37),
\end{aligned}
$$

$\longrightarrow$ on $K:(16,24,33,40,51,54,58)$ (by domination). For $\mathbf{L}$

$$
\begin{aligned}
G \oplus \mathrm{GL} & =(3,9,15,20,28,30,32) \oplus(3,5,6,7,8,9,10) \\
& =(6,14,21,27,36,39,42), \\
H \oplus \mathrm{HL} & =(2,3,4,8,13,14,15) \oplus(3,4,5,17,19,21,22) \\
& =(5,7,9,25,31,35,37),
\end{aligned}
$$

$\longrightarrow$ on $L:(6,14,21,27,36,39,42)$ (by domination).

For $\mathbf{M}$

$$
\begin{aligned}
I \oplus \mathrm{IM}= & (4,10,15,26,30,32,35) \oplus(6,6,6,6,7,7,7) \\
= & (10,16,21,32,37,39,42) \text { gives } \\
& \cdot \frac{(10+16+21+66+37+39+42)}{8}=28.625, \\
E \oplus \mathrm{EM}= & (1,3,6,13,15,16,17) \oplus(5,10,15,22,23,24,25) \\
= & (6,13,21,35,38,40,42) \text { gives } \\
& \cdot \frac{(6+13+21+70+38+40+42)}{8}=28.75, \\
& \longrightarrow \text { on } M:(6,13,21,35,38,40,42) .
\end{aligned}
$$

For $\mathbf{N}$

$$
\begin{aligned}
K \oplus \mathrm{KN} & =(16,24,33,40,51,54,58) \oplus(6,7,8,9,10,11,12) \\
& =(22,31,41,49,61,65,70), \\
L \oplus \mathrm{LN} & =(6,14,21,27,36,39,42) \oplus(2,3,4,5,6,7,8) \\
& =(8,17,25,32,42,46,50), \\
F \oplus \mathrm{FN} & =(1,2,6,12,13,14,15) \oplus(1,3,4,5,7,8,10) \\
& =(2,5,10,17,20,22,25), \\
J \oplus \mathrm{JN} & =(4,7,11,21,23,26,29) \oplus(6,7,8,9,10,11,12) \\
& =(10,14,19,30,33,37,41), \\
M \oplus \mathrm{MN} & =(6,13,21,35,38,40,42) \oplus(7,8,9,11,12,13,14) \\
& =(13,21,30,46,50,53,56),
\end{aligned}
$$

$\longrightarrow$ on $N:(22,31,41,49,61,65,70)$ (by domination).
Proceeding backward using the Minkowski's subtraction in Definition 8 to find the critical path as follows:

$$
\begin{aligned}
& \underset{m}{N(-)} \mathrm{KN}=(22,31,41,49,61,65,70) \underset{m}{(-)} \\
& \cdot(6,7,8,9,10,11,12) \\
& =(16,24,33,40,51,54,58) \\
& =(16,24,33,40,51,54,58) \text {, } \\
& \underset{m}{N(-)} \mathrm{LN}=(22,31,41,49,61,65,70) \underset{m}{(-)} \\
& \cdot(2,3,4,5,6,7,8) \\
& =(20,28,37,44,55,58,62) \\
& \neq(6,14,21,27,36,39,42) \text {, } \\
& \underset{m}{N(-)} \mathrm{FN}=(22,31,41,49,61,65,70) \underset{m}{(-)} \\
& \cdot(1,3,4,5,7,8,10) \\
& =(21,28,37,44,54,57,60) \\
& \neq(1,2,6,12,13,14,15) \text {, } \\
& \underset{m}{N(-)} \mathrm{JN}=(22,31,41,49,61,65,70) \underset{m}{(-)} \\
& \cdot(6,7,8,9,10,11,12) \\
& =(16,24,33,40,51,54,58) \\
& \neq(4,7,11,21,23,26,29) \text {, } \\
& \underset{m}{N(-)} \mathrm{MN}=(22,31,41,49,61,65,70) \underset{m}{(-)} \\
& \cdot(7,8,9,11,12,13,14) \\
& =(15,23,32,38,49,52,56) \\
& \neq(6,13,21,35,38,40,42) \text {. }
\end{aligned}
$$

Therefore, the critical path passes through $K$

$$
\begin{aligned}
\underset{m}{K(-)} \mathrm{GK}= & (16,24,33,40,51,54,58) \underset{m}{(-)} \\
& \cdot(13,15,18,20,23,24,26) \\
= & (3,9,15,20,28,30,32)=(3,9,15,20,28,30,32), \\
\underset{m}{K(-)} \mathrm{BK}= & (16,24,33,40,51,54,58)(-) \\
& \cdot(3,4,5,11,19,20,22) \\
= & (13,20,28,29,32,34,36) \neq(2,3,4,8,13,14,15) .
\end{aligned}
$$

The critical path passes through $G$.

$$
\begin{aligned}
\underset{m}{G(-) B G} & =(3,9,15,20,28,30,32) \underset{m}{(-)}(1,6,11,12,15,16,17) \\
& =(2,3,4,8,13,14,15), \\
\underset{m}{G(-)} \mathrm{CG} & =(3,9,15,20,28,30,32) \underset{m}{(-)}(2,3,4,8,14,15,16) \\
& =(1,6,7,12,14,15,16) \neq(2,3,4,8,13,14,15) .
\end{aligned}
$$


The critical path passes through $B$. Therefore, the critical path has the vertices $(A, B, G, K, N)$, and the pessimistic data is $(22,31,41,49,61,65,70)$.

The optimization process with the data given in Table 2 is as follows:

$$
\begin{aligned}
& \longrightarrow \text { on } B: 8.375, \\
& \longrightarrow \text { on } C: 6.875, \\
& \longrightarrow \text { on } D: 5.5, \\
& \longrightarrow \text { on } E: 10.5, \\
& \longrightarrow \text { on } F: 9.375 .
\end{aligned}
$$

For $\mathbf{G}$

$$
\begin{aligned}
B+\mathrm{BG} & =8.375+11.25=19.625, \\
C+\mathrm{CG} & =6.875+8.75=15.625, \\
& \longrightarrow \text { on } G: 19.625 .
\end{aligned}
$$

For $\mathbf{H}$

$$
\begin{gathered}
D+\mathrm{DH}=5.5+4.5=10, \\
\longrightarrow \text { on } H: 10 .
\end{gathered}
$$

For $\mathbf{I}$

$$
\begin{aligned}
D+\mathrm{DI} & =5.5+6.125=11.625, \\
E+\mathrm{EI} & =10.5+11.75=22.25, \\
& \longrightarrow \text { on } I: 22.25 .
\end{aligned}
$$

For J

$$
\begin{aligned}
C+\mathrm{CJ}= & 6.875+10.875=17.75, \\
& \longrightarrow \text { on } J: 17.75 .
\end{aligned}
$$

For K

$$
\begin{aligned}
G+\mathrm{GK} & =19.625+19.875=39.5, \\
B+\mathrm{BK} & =8.375+11.875=20.25, \\
& \longrightarrow \text { on } K: 39.5 .
\end{aligned}
$$

For $\mathbf{L}$

$$
\begin{aligned}
G+\mathrm{GL} & =19.625+6.875=26.5 \\
H+\mathrm{HL} & =10+11.125=21.125, \\
& \longrightarrow \text { on } L: 26.5
\end{aligned}
$$

For $\mathbf{M}$

$$
\begin{aligned}
I+\mathrm{IM} & =22.25+6.375=28.625 \\
E+\mathrm{EM} & =10.5+18.25=28.75 \\
& \longrightarrow \text { on } M: 28.75
\end{aligned}
$$

For $\mathbf{N}$

$$
\begin{gathered}
K+\mathrm{KN}=39.5+9=48.5, \\
L+\mathrm{LN}=26.5+5=31.5, \\
F+\mathrm{FN}=9.375+5.375=14.75, \\
J+\mathrm{JN}=17.75+5.75=23.5, \\
M+\mathrm{MN}=28.75+10.625=39.375, \\
\longrightarrow \text { on } N: 48.5 .
\end{gathered}
$$

The critical path is $(A, B, G, K, N)$, and the associated ordinary number is 48.5 .

For the associated ordinary numbers, the floating times merger can be computed for the vertices as follows:

For $\mathbf{L}$

$$
\begin{gathered}
N-\mathrm{LN}=48.5-5=43.5, \\
N-\mathrm{LN}-L=43.5-26.5=17,
\end{gathered}
$$

$\longrightarrow$ Floating time at $L: 17$.

For $\mathbf{F}$

$$
\begin{gathered}
N-\mathrm{FN}=48.5-5.375=43.125, \\
N-\mathrm{FN}-F=43.125-9.375=33.75,
\end{gathered}
$$

$\longrightarrow$ Floating time at $F: 33.75$.

For $\mathbf{M}$

$$
\begin{gathered}
N-\mathrm{MN}=48.5-10.625=37.875, \\
N-\mathrm{MN}-M=37.875-28.75=9.125, \\
\longrightarrow \text { Floating time at } M: 9.125 .
\end{gathered}
$$

To compute the other floating times, one proceeds backward taking the smallest floating time as follows:

For $\mathbf{H}$

$$
\begin{gathered}
L-\mathrm{HL}=N-\mathrm{LN}-\mathrm{HL}=48.5-5-11.125=32.375, \\
L-\mathrm{HL}-H=37.875-10=27.875 \\
\quad \longrightarrow \text { Floating time at } H: 27.875 .
\end{gathered}
$$


Table 3: Optimistic H. F.N. for each operation from $A$ to $N$ and corresponding associated numbers.

\begin{tabular}{lcc}
\hline Path & H.F.N. $A_{H \circ}$ & $\widehat{A}_{H} \circ$ \\
\hline $1 \mathrm{AB}$ & $(3,4,5,7,9,10,11)$ & 7 \\
$2 \mathrm{AC}$ & $(0,1,3,4,12,13,14)$ & 6.375 \\
$3 \mathrm{AD}$ & $(0,1,2,4,6,7,8)$ & 4 \\
$4 \mathrm{AE}$ & $(1,2,4,10,11,12,13)$ & 7.875 \\
$5 \mathrm{AF}$ & $(0,1,2,8,10,13,14)$ & 7 \\
$6 \mathrm{BG}$ & $(1,2,7,8,9,10,13)$ & 7.25 \\
$7 \mathrm{BK}$ & $(2,5,8,10,11,12,13)$ & 8.875 \\
$8 \mathrm{CG}$ & $(1,3,5,8,9,10,11)$ & 6.875 \\
$9 \mathrm{CJ}$ & $(1,2,5,9,10,12,14)$ & 7.75 \\
$10 \mathrm{DH}$ & $(0,1,2,3,4,5,7)$ & 3.125 \\
$11 \mathrm{DI}$ & $(1,2,3,4,5,7,8)$ & 4.25 \\
$12 \mathrm{EI}$ & $(2,5,8,10,11,12,13)$ & 8.875 \\
$13 \mathrm{EM}$ & $(3,6,8,10,11,12,13)$ & 9.125 \\
$14 \mathrm{FN}$ & $(0,1,2,3,4,5,6)$ & 3 \\
$15 \mathrm{GK}$ & $(7,10,13,16,17,18,19)$ & 14.5 \\
$16 \mathrm{GL}$ & $5,5,5,5,5,5,5$ & 5 \\
$17 \mathrm{HL}$ & $(5,7,8,9,10,11,12)$ & 8.875 \\
$18 \mathrm{IM}$ & $(5,5,5,5,5,5,5)$ & 5 \\
$19 \mathrm{JN}$ & $(1,3,4,5,6,7,8)$ & 8.875 \\
$20 \mathrm{KN}$ & $(3,4,5,6,7,8,9)$ & 6 \\
$21 \mathrm{LN}$ & $(0,1,2,3,4,5,6)$ & \\
$22 \mathrm{MN}$ & $(5,6,7,8,9,10,11)$ & \\
\hline & & \\
& &
\end{tabular}

For $\mathbf{I}$

$M-\mathrm{IM}=N-\mathrm{MN}-\mathrm{IM}=48.5-10.625-6.375=31.5$, $M-\mathrm{IM}-I=31.5-22.25=9.25$,

$\longrightarrow$ Floating time at $I: 9.25$.

For $\mathbf{J}$

$$
\begin{gathered}
N-\mathrm{JN}=48.5-5.75=42.75, \\
N-\mathrm{JN}-J=42.75-17.75=25,
\end{gathered}
$$

$\longrightarrow$ Floating time at $J: 25$.

For $\mathbf{C}$

$$
\begin{gathered}
G-\mathrm{CG}=19.625-8.75=10.875, \\
G-\mathrm{CG}-C=10.875-8.75=2.125, \\
\longrightarrow \text { Floating time at } C: 2.125 .
\end{gathered}
$$

\section{For D}

$$
\begin{gathered}
H-\mathrm{DH}=N-\mathrm{LN}-\mathrm{HL}-\mathrm{DH}=48.5-5 \\
-11.125-4.5=27.875 \\
H-\mathrm{DH}-\mathrm{D}=27.875-5.5=22.375 \\
I-\mathrm{DI}=N-\mathrm{MN}-\mathrm{IM}-\mathrm{DI}=48.5-10.625 \\
\quad-6.375-6.125=25.375 \\
I-\mathrm{DI}-D=25.375-5.5=19.875 \\
\quad \text { Floating time at } D: 19.875
\end{gathered}
$$

For E

$$
\begin{gathered}
I-\mathrm{EI}=N-\mathrm{MN}-\mathrm{IM}-\mathrm{EI}=48.5-10.625 \\
\quad-6.375-11.75=19.75 \\
I-\mathrm{EI}-E=19.75-10.5=9.25 \\
M-\mathrm{EM}=\mathrm{N}-\mathrm{MN}-\mathrm{EM}=48.5-10.625 \\
\quad-18.25=19.625 \\
M-\mathrm{EM}-E=19.625-10.5=9.125, \\
\longrightarrow \text { Floating time at } E: 9.125 .
\end{gathered}
$$

For $\mathbf{F}$

$$
\begin{gathered}
N-\mathrm{FN}=48.5-5.375=43.125, \\
N-\mathrm{FN}-F=43.125-9.375=33.75,
\end{gathered}
$$

$\longrightarrow$ Floating time at $F: 33.75$.

Other kinds of margins may be used as in classical CPM methods.

4.2. Optimistic Critical Path Computations. We shall now repeat the computations for the optimistic case. A table will be computed first which corresponds to the minor H.F.N.s. of Table 1. The minors, which are associated with ordinary numbers, are tabulated in Table 3.

For this purpose, we shall not present the computations using the H.F.N.s. as we did with the pessimistic data. So, we carry out these computations from what has been given already. Using the associated ordinary numbers for optimistic data from Table 3, the optimization process gives

$$
\begin{aligned}
& \longrightarrow \text { on } B: 8.375, \\
& \longrightarrow \text { on } C: 6.875, \\
& \longrightarrow \text { on } D: 5.5, \\
& \longrightarrow \text { on } E: 10.5, \\
& \longrightarrow \text { on } F: 9.375
\end{aligned}
$$


For $\mathbf{G}$

$$
\begin{aligned}
B+\mathrm{BG} & =8.375+7.25=15.625 \\
C+\mathrm{CG} & =6.875+6.875=13.75 \\
& \longrightarrow \text { on } G: 15.625
\end{aligned}
$$

For $\mathbf{H}$

$$
\begin{aligned}
D+\mathrm{DH} & =5.5+3.125=8.625, \\
\longrightarrow & \text { on } H: 8.625 .
\end{aligned}
$$

For I

$$
\begin{gathered}
D+\mathrm{DI}=5.5+4.25=9.75 \\
E+\mathrm{EI}=10.5+8.875=19.375 \\
\longrightarrow \text { on } I: 19.375 .
\end{gathered}
$$

For $\mathbf{J}$

$$
\begin{aligned}
C+\mathrm{CJ} & =6.875+7.75=14.625, \\
& \longrightarrow \text { on } J: 14.625 .
\end{aligned}
$$

For $\mathbf{K}$

$$
\begin{aligned}
G+\mathrm{GK} & =15.625+14.5=30.125, \\
B+\mathrm{BK} & =8.375+8.875=17.25, \\
& \longrightarrow \text { on } K: 30.125 .
\end{aligned}
$$

For $\mathbf{L}$

$$
\begin{aligned}
G+\mathrm{GL} & =15.625+5=20.625 \\
H+\mathrm{HL} & =8.625+8.875=17.50 \\
& \longrightarrow \text { on } L: 20.625
\end{aligned}
$$

For M

$$
\begin{aligned}
I+\mathrm{IM} & =19.375+5=24.625 \\
E+\mathrm{EM} & =10.5+9.125=19.625 \\
& \longrightarrow \text { on } M: 24.625
\end{aligned}
$$

For $\mathbf{N}$

$$
\begin{gathered}
K+\mathrm{KN}=30.125+6=36.125, \\
L+\mathrm{LN}=20.625+3=23.625, \\
F+\mathrm{FN}=9.375+3=12.375, \\
J+\mathrm{JN}=14.625+4.875=19.5, \\
M+\mathrm{MN}=24.625+8=32.625, \\
\longrightarrow \text { on } N: 36.125
\end{gathered}
$$

Applying the progressing backward to look for the optimistic critical path as

$$
\begin{gathered}
N-\mathrm{KN}=36.125-6=30.125=K=30.125, \\
N-\mathrm{LN}=36.125-3=33.125 \neq L=20.625, \\
N-\mathrm{FN}=36.125-3=33.125 \neq F=9.375, \\
N-\mathrm{JN}=36.125-4.875=31.25 \neq J=14.625, \\
N-\mathrm{MN}=36.125-8=28.125 \neq M=24.625
\end{gathered}
$$

The critical path passes through $K$

$$
\begin{aligned}
& K-\mathrm{GK}=30.125-14.5=15.625=G=15.625, \\
& K-\mathrm{BK}=36.125-7.25=28.875 \neq B=8.375, \\
& \text { and passing } G \\
& G-\mathrm{BG}=15.625-7.25=8.375=B=8.375, \\
& G-\mathrm{CG}=15.625-6.875=8.75 \neq C=6.875
\end{aligned}
$$

It also path through $B$, and we have, therefore, the optimistic critical path $(A, B, G, K, N)$ with the associated ordinary number 36.125.

It is observed that in this case the optimistic critical path is the same as the pessimistic one. This is an unusual case, and using other data different critical paths may be found.

Now, we will calculate the floating times for vertices, which are not on the critical path.

For $\mathbf{L}$

$$
\begin{gathered}
N-\mathrm{LN}=36.125-3=33.125, \\
N-\mathrm{LN}-L=33.125-20.625=12.50, \\
\longrightarrow \text { Floating time at } L: 12.50 .
\end{gathered}
$$

For $\mathbf{F}$

$$
\begin{gathered}
N-\mathrm{FN}=36.125-3=33.125, \\
N-\mathrm{FN}-F=33.125-9.375=23.75, \\
\longrightarrow \text { Floating time at } F: 23.75 .
\end{gathered}
$$

For $\mathbf{J}$

$$
\begin{gathered}
N-\mathrm{JN}=36.125-4.875=31.25, \\
N-\mathrm{JN}-J=31.25-14.625=16.625, \\
\longrightarrow \text { Floating time at } J: 16.625 .
\end{gathered}
$$

For $\mathbf{M}$

$$
\begin{gathered}
N-\mathrm{MN}=36.125-8=28.125, \\
N-\mathrm{MN}-M=28.125-24.625=3.5, \\
\longrightarrow \text { Floating time at } M: 3.5 .
\end{gathered}
$$


For $\mathbf{H}$

$$
\begin{gathered}
N-\mathrm{HL}=N-\mathrm{LN}-\mathrm{HL}=36.125-3-8.875=24.25, \\
N-\mathrm{HL}-H=24.25-8.625=15.625,
\end{gathered}
$$

$\longrightarrow$ Floating time at $H: 15.625$.

For I

$$
\begin{gathered}
M-\mathrm{IM}=N-\mathrm{MN}-\mathrm{IM}=36.125-8-5=23.125, \\
N-\mathrm{IM}-I=23.125-19.375=3.75, \\
\longrightarrow \text { Floating time at } I: 3.75 .
\end{gathered}
$$

For J

$$
\begin{gathered}
N-\mathrm{JN}=36.125-4.875=31.25, \\
N-\mathrm{JN}-J=31.25-16.625=14.625, \\
\longrightarrow \text { Floating time at } J: 14.625 .
\end{gathered}
$$

For C

$$
\begin{gathered}
G-\mathrm{CG}=15.625-6.875=8.75, \\
C-\mathrm{CG}-C=8.75-6.875=1.875, \\
\longrightarrow \text { Floating time at } C: 1.875 .
\end{gathered}
$$

For D

$$
\begin{gathered}
H-\mathrm{DH}=N-\mathrm{LN}-\mathrm{HL}-\mathrm{DH}=36.125 \\
-3-8.875-3.125=21.125, \\
H-\mathrm{DH}-D=21.125-5.5=15.625, \\
I-\mathrm{DI}=N-\mathrm{MN}-\mathrm{IM}-\mathrm{DI}=36.125 \\
\quad-8-5-4.25=18.875, \\
I-\mathrm{DI}-D=18.875-5.5=13.375, \\
\longrightarrow \text { Floating time at } D: 13.375 .
\end{gathered}
$$

For $\mathbf{E}$

$$
\begin{gathered}
I-\mathrm{EI}=N-\mathrm{MN}-\mathrm{EI}-8.875=36.125 \\
\quad-8-5-8.875=14.25, \\
I-\mathrm{EI}-E=14.25-10.5=3.75, \\
M-\mathrm{EM}=\mathrm{N}-\mathrm{MN}-\mathrm{EM}=36.125-8-9.125=19, \\
M-\mathrm{EM}-E=19-10.5=8.5, \\
\longrightarrow \text { Floating time at } E: 13.375 .
\end{gathered}
$$

For $\mathbf{F}$

$$
\begin{gathered}
N-\mathrm{FN}=36.125-3=33.125, \\
N-\mathrm{FN}-F=33.125-9.375=23.75, \\
\longrightarrow \text { Floating time at } F: 23.75 .
\end{gathered}
$$

\section{Discussion of the Results}

It is noted that the floating times with optimistic data are always smaller than or equal to the corresponding floating times for the pessimistic data. This is the consequence of a contraction in the whole network. It is logical to consider that the margins decrease when the operative data decreases.

The process which is characterized by uncertain and subjective data can be summarized as follows:

(i) Experts must be selected for each operation; the number selected being determined by the type of operation and the uncertainty encountered. There is no priori rule for their selection. Each expert, however, must give his/her subjective opinion in the form of H.F.N

(ii) For each operation, a major H.F.N. and a minor H.F.N. are selected. Of course, if only one H.F.N. exists, it is own major and minor. For some operations, the H.F.N.s. are just ordinary numbers

(iii) For each major H.F.N. and minor H.F.N., the associated ordinary number must be computed

(iv) To determine the pessimistic critical path, the major H.F.N.s. and their associated ordinary numbers are employed. For optimistic critical path, minor H.F.N.s. and their associated ordinary numbers are employed

(v) The floating times are then calculated using associated ordinary numbers for vertices which are not on the critical path

(vi) Following these calculations, the experts reevaluate their H.F.N. estimates, and the process is repeated

In addition, in the illustrative example, four experts established the data, but in a nonacademic example, this number depends on many factors: available experts, kind of operation, cost of operation, and so on. However, it appears that the computations of the major H.F.N. and the minor H.F.N. are very useful in many realistic situations.

\section{Conclusions}

In this paper, method for solving litigation problems by experts when they share the same information but differ in their opinions has been introduced. An example with data has been established by four experts, but in a nonacademic example, this number depends on many factors: available experts, kind of operation, cost of operation, and so on. It 
has appeared the computations of the major and minor of H.F.N. are useful in many realistic situations.

The proposed model is also applicable to more complicated project networks in real world. For future research, the neutrosophic sets and interval type can be considered. In addition, the procedure can be modified depending on the nature of the analysis, and the process described is useful especially when the opinions of many experts are necessary as may be required in a large scale project.

\section{Data Availability}

The data used to support the findings of this research are available from the corresponding author upon request.

\section{Disclosure}

Hamiden Abd El-Wahed Khalifa present address is Mathematics Department, College of Science and Arts, Al-Badaya, Qassim University, Saudi Arabia.

\section{Conflicts of Interest}

The authors do not have any conflicts of interest.

\section{References}

[1] K. J. Bassey and P. E. Chigbu, "On building a OR-based decision model via coherent pluralism," Global Journal of Pure and Applied Sciences, vol. 8, no. 3, pp. 347-355, 2002.

[2] A. M. Aliyu, "Project management using critical path method (CPM): a pragmatic study," Global Journal of Pure and Applied Sciences, vol. 18, no. 3-4, pp. 197-206, 2013.

[3] W. Agyei, "Project planning and scheduling using PERT and CPM techniques with linear programming," International Journal of Scientific \& Technology Research, vol. 4, no. 8, pp. 2277-8616, 2015.

[4] M. S. Rautela, A. Pandey, S. Garg, and P. Damle, "Case study: making and monitoring of critical path in shoe production planning," Journal of Business Management \& Social Science Research, vol. 4, no. 3, pp. 2319-5614, 2015.

[5] W.-M. Chu, K. Y. Chang, C. Y. Lu, C. H. Hsu, C. H. Liu, and Y. C. Hsiao, "A new approach to determine the critical path in stochastic activity network," Mathematical Problems in Engineering, vol. 2014, Article ID 547627, 13 pages, 2014.

[6] L. A. Zadeh, "Fuzzy sets," Information and Control, vol. 8, no. 3, pp. 338-353, 1965.

[7] R. E. Bellman and L. A. Zadeh, "Decision making in a fuzzy environment," Decision Sciences, vol. 17, no. 4, pp. B-141-B164, 1970.

[8] H. J. Zimmermann, "Fuzzy programming and linear programming with several objective functions," Fuzzy Sets and System, vol. 1, no. 1, pp. 45-55, 1978.

[9] D. Dubois and H. Prade, Fuzzy Sets and Systems: Theory and Applications, Academic Press, New York, NY, USA, 1980.

[10] A. Kaufmann and M. M. Gupta, Fuzzy Mathematical Models in Engineering and Management Science, Elsevier Science Publishing Company INC, New York, NY, USA, 1988.

[11] S. Chanas and P. Zielinski, "Critical path analysis in the network with fuzzy activity times," Fuzzy Sets and Systems, vol. 122, no. 2, pp. 195-204, 2001.
[12] F. Arikan and Z. Gungor, "An application of fuzzy goal programming to a multiobjective project network problem," Fuzzy Sets and Systems, vol. 119, no. 1, pp. 49-58, 2001.

[13] G. S. Liang and T. C. Han, "Fuzzy critical path for project network," Information and Management Science, vol. 15, no. 4, pp. 29-40, 2004.

[14] P. Zielinkski, "On computing the latest starting times and floats of activities in a network with imprecise durations," Fuzzy Sets and Systems, vol. 150, no. 1, pp. 53-76, 2005.

[15] S. P. Chen, "Analysis of critical paths in a project network with fuzzy activity times," European Journal of Operational Research, vol. 183, no. 1, pp. 442-459, 2007.

[16] S. P. Chen and Y. J. Hsueh, "A simple approach to fuzzy critical path analysis in project networks," Applied Mathematical Modelling, vol. 32, no. 7, pp. 1289-1297, 2008.

[17] M. Ghazanfari, A. Yousefli, M. S. Jabal Ameli, and A. BozorgiAmiri, "A new approach to solve time-cost trade-off problem with fuzzy decision variables," International Journal of manufacturing Technology, vol. 42, no. 3-4, pp. 408-414, 2009.

[18] N. R. Shankar, V. Sireesha, and P. B. Rao, "An analytical method for finding critical path in a fuzzy project network," International Journal of Contemporary Mathematical Sciences, vol. 5, no. 20, pp. 953-962, 2010.

[19] T. F. Liang, “Applying fuzzy goal programming to project management decisions with multiple goals in uncertain environments," Expert Systems with Applications, vol. 37, no. 12, pp. 8499-8507, 2010.

[20] V. Sireesha and N. R. Shankar, "A new approach to find total float time and critical path in a fuzzy project network," International Journal of Engineering Science and Technology, vol. 2, no. 4, pp. 600-609, 2010.

[21] M. Ammar, "Optimization of project time-cost trade-off problem with discounted cash flows," Journal of Construction Engineering \& Management, vol. 137, no. 1, pp. 65-71, 2011.

[22] N. Shahsavari Pour, M. Kheranmand, M. Fallah, and S. Zeynali, "A new method for critical path method with fuzzy processing time," Management Science Letters, vol. 1, no. 3, pp. 347-354, 2011.

[23] S. Elizabeth and L. Sujatha, "Fuzzy critical path problem for project network," International Journal of Pure and Applied Mathematics, vol. 85, no. 2, pp. 223-240, 2013.

[24] W. S. Khalaf, "Solving the fuzzy project scheduling problem based on a ranking function," Australian Journal of Basic and Applied Sciences, vol. 7, no. 8, pp. 806-811, 2013.

[25] M. H. Oladeinde and D. O. Itsisor, "Application of fuzzy theory to project scheduling with critical path method," Journal of Applied Sciences and Environmental Management, vol. 17, no. 1, pp. 161-166, 2013.

[26] V. Anusuya and P. Balasowandari, "Fuzzy critical path with various measures," International Journal of Mathematics Trends and Technology, vol. 54, no. 5, pp. 366-370, 2018.

[27] Jin-Shing Yao and Feng-Tse Lin, "Fuzzy critical path method based on signed distance ranking of fuzzy numbers," IEEE Transactions on Systems, Man, and Cybernetics - Part A: Systems and Humans, vol. 30, no. 1, pp. 76-82, 2000.

[28] D. Dutta and P. Kumar, "Application of fuzzy goal programming approach to multi-objective linear fractional inventory model," International Journal of Systems Science, vol. 46, no. 12, pp. 2269-2278, 2015.

[29] H. Abd el-Wahed Khalifa, P. Kumar, and M. G. Alharbi, "Enhancement of capacitated transportation problem in fuzzy 
environment," Advances in Fuzzy Systems, vol. 2020, Article ID 8893976, 9 pages, 2020.

[30] H. Abd el-Wahed Khalifa, S. S. Alodhaibi, and P. Kumar, "Solving constrained flow-shop scheduling problem through multistage fuzzy binding approach with fuzzy due dates," Advances in Fuzzy Systems, vol. 2021, Article ID 6697060, 8 pages, 2021.

[31] P. Selvam, A. Rajkumar, and J. S. Easwari, "Fuzzy assignment problems using normalized heptagonal fuzzy numbers," International Journal of Pure and Applied Mathematics, vol. 117, no. 11, pp. 405-415, 2017.

[32] P. K. Usha and P. Kumar, "Single transmit fuzzy queuing model with two-classes: execution proportions by ranking technique," Journal of Engineering Science and Technology, vol. 15, no. 4, pp. 2395-2406, 2020.

[33] S. Radhakrishnan and D. Saikeerthana, "Research article interval network analysis in project management," Kongunadu Research Journal, vol. 7, no. 2, pp. 99-113, 2020. 